\title{
Enseñanza de la geometría en población invidente y de baja visión ${ }^{1}$
}

\author{
Teaching blind population geometry and low vision \\ Ensinar geometria população cega e baixa visão
}

Recibido: mayode 2013

Aceptado: agosto de 2013
Miguel Ángel Niño Angarita ${ }^{2}$

Lina Fernanda Vanegas Gutiérrez ${ }^{3}$

\section{Resumen}

En este artículo se realizó un corto análisis acerca de una actividad planeada para el área de matemáticas, específicamente dentro del ámbito geométrico el cual tiene como finalidad la enseñanza de la geometría dentro de la diversidad del aula. Por ello surge esta propuesta dentro del contorno del aula inclusiva, caso de las matemáticas como área excluyente, para determinar las relaciones particulares de las poblaciones con discapacidad visual con los distintos contenidos y destrezas matemáticas en el contexto escolar ordinario.

Palabras clave: Aula inclusiva; alumno; necesidades especiales; alumnos discapacitados; diversidad; matemáticas escolares; geometría; enseñanza de la geometría.

\begin{abstract}
This article made a short analysis of a planned activity for the area of mathematics, specifically within the geometric field which is aimed at teaching geometry in classroom diversity. So does this proposal within the inclusive classroom contour case of mathematics as exclusive area to determine the particular relationships of visually impaired people with different contents and math skills in the regular school setting.
\end{abstract}

Keywords: Inclusive classroom, student, special needs, students with disabilities, diversity, school mathematics, geometry, teaching geometry.

\section{Resumo}

Este artigo fez uma breve análise de uma atividade planejada para a área da matemática, especificamente dentro do campo geométrico que se destina a ensinar geometria na diversidade em sala de aula. $O$ mesmo acontece com esta proposta no caso, inclusive o contorno da sala de aula de matemática como área exclusiva para determinar as relações específicas das pessoas com deficiência visual com diferentes conteúdos e habilidades matemáticas no ambiente escolar regular.

1 Artículo de Investigación.

2 Universidad Distrital Francisco José de Caldas. Bogotá, Colombia. Contacto: miguelangel@hotmail.com

3 Universidad Distrital Francisco José de Caldas. Bogotá, Colombia. Contacto: linita-monis@hotmail.com 
Palavras-chave: Sala de aula inclusiva, estudante, necessidades especiais, alunos com deficiência, a diversidade, a matemática da escola, geometria, geometria ensino.

\section{Presentación del problema}

En este documento surgió en primera instancia acerca de la concepción de la didáctica de las matemáticas aplicadas en la enseñanza-aprendizaje a poblaciones invidentes, es por ello que se pretendió tener en cuenta la diversidad dentro del aula de matemáticas y el cómo se van vinculando las necesidades educativas especiales (NEE), partiendo de que la enseñanza es más caracterizada y personalizada, de tal forma que se necesita de una atención diferenciada; por ende la profesión docente debe planear diversas estrategias para llevar a cabo la enseñanza de las matemáticas en este caso de la geometría, por el componente visual que comporta.

\section{Marco de referencia conceptual}

En la educación matemática se encuentran inmersas, diferentes actividades para llevar a cabo un aprendizaje significativo en las matemáticas, es por ello que se quiere mencionar principalmente la enseñanza de la geometría y la concepción de los docentes hacia está, ya que casi siempre se ha evidenciado en las prácticas pedagogías, que muchos de los docentes relacionan la geometría, específicamente con temáticas como figuras o dibujos, nombres, definición, superficies, volúmenes y perímetros, restringiendo la geometría a discutas netamente métricas y conllevando a la clase en un listado geométrico. Consecutivamente se hace mención al método de resolución de problemas, ya que estos promueven la enseñanza de las matemáticas y de igual forma permite que el estudiante construya su propio conocimiento a través de la guía y orientación del docente, de tal forma que en la geometría involucre el uso de relaciones y conceptos del pensamiento geométrico.
Ahora bien, si se tiene en cuenta la enseñanza de la geometría a poblaciones con discapacidad visual, se debe identificar de manera práctica cuáles van hacer las estrategias, habilidades, conocimientos y dificultades específicas de esta población en comparación con sujetos videntes y de esta forma poder realizar una adaptación curricular del área de matemáticas, específicamente de la geometría. De tal forma, dentro de la orientación de las didácticas de las matemáticas se ha determinado una conceptualización del conocimiento, en otras palabras es englobar todo lo que el sujeto es capaz de decir o hacer en relación a un objeto (Chevallard, SF), lo anterior conlleva a caracterizar los contenidos geométricos elementales, tal como afirma (Forte \& Garcia) Godino y Batanero (2003), que por medio de la interpretación semiótica en la noción relación al objeto, se podría caracterizar los contenidos geométricos. (Godino \& Batanero, 2003)

En correspondencia con lo dicho anteriormente, cabe mencionar que las personas con discapacidad visual, se les obstaculiza caracterizar los diversos contenidos geométricos, ya que mediante la información espontánea del medio queda reducida, deformada y necesita de otros sentidos como el oído, el tacto o el olfato o de la información que pueden suministrar otras personas (Equipo de Apoyo Educativo a Ciegos y Deficientes Visuales, $\mathrm{SF}$ ), en este caso los docentes del área de matemáticas, cuyo objetivo es permitirles conocer el entorno por medio de las habilidades de los estudiantes, ya que estos logran construir el conocimiento acerca del medio que lo rodea, es decir básicamente a través de los estímulos visuales.

Por último se tiene en cuenta la enseñanza de la geometría a poblaciones invidentes, teniendo como fundamento que el estudiante por medio del tacto va a explorar, aprender y sobre todo disfrutar de la actividad propuesta, es así que el docente debe realizar una adaptación de las representaciones 
graficas-visuales-textuales y hacerlas palpables dentro de un contexto real, permitiendo que el estudiante comprenda y asimile la información presentada, de este modo lograr que el estudiante adquiera los conocimientos de una forma más clara y a través de su exploración con el recurso manipulativo llegue a interiorizar los objetivos propuestos para las actividades de matemáticas.

\section{Metodología}

Esta investigación es de carácter experimental-descriptivo, que consiste en conocer las situaciones y costumbres predominantes a través de las mismas haciendo una descripción exacta de la actividad, la intención no es sólo recolectar datos, además se tienen en cuenta las relaciones que existen entre determinadas variables, basándose en alguna teoría, recogen y exponen la información para analizarla cuidadosamente con el fin de extraer conclusiones significativas que aporten al conocimiento (Deobold, Van, \& Meyer, 2006)

Aplicación. Se eligió una población de estudiantes de grado $6^{\circ}$ de la básica secundaria del colegio José Félix Restrepo, con el motivo de analizar el grado de dificultad en cuanto la enseñanza de la geometría.

La muestra consta de: Estudiantes invidentes (5); Estudiante videntes (5); Estudiantes con los ojos tapados (5).

Cuando se pasó el cuestionario se insistió a los estudiantes que no se trataba de una actividad valorativa, sino de un estudio para manifestar los problemas de aprendizaje de la geometría en general y, particularmente, en el caso de la población de estudiantes con discapacidad visual, para intentar aportar alguna solución con el fin de disminuir la dificultad de dicho aprendizaje, logrando permitir que los estudiantes se sintieran partícipes de la investigación.
Les pedimos que contestasen a todas las preguntas con el máximo interés ya que, a pesar de los posibles fallos, sus profesores no lo iban a tener en cuenta al calificarlos.

\section{Análisis de datos}

Se realizó una primera disertación del cuestionario analizando cada uno de los puntos propuestos, según el mecanismo temático para los tres grupos de estudiantes, una segunda disertación fue a través de los contenidos y un tercer análisis según los caracteres asignados a cada pregunta obteniéndose las siguientes conclusiones:

- La percepción visual influye preclaramente en la representación. Estudiantes Invidentes (28\%), Estudiantes Videntes (76\%), Estudiantes con los ojos Tapados (45\%)

- La percepción visual también influye la representación métrica. Estudiantes Invidentes (20\%), Estudiantes Videntes (58\%), Estudiantes con los ojos Tapados $(46 \%)$

- En la clasificación de las diferencias entre videntes e invidentes es poco significativa. Estudiantes Invidentes 68\%), Estudiantes Videntes (90\%), Estudiantes con los ojos Tapados (91\%)

- A la hora de entender un concepto los invidentes logran mayor nivel de éxito. Estudiantes Invidentes (89\%), Estudiantes Videntes (45\%), Estudiantes con los ojos Tapados (44\%)

- A la hora de construir cuerpos geométricos los invidentes obtienen resultados inferiores a los de los otros grupos. Estudiantes Invidentes (30\%), Estudiantes Videntes (100\%), Estudiantes con los ojos Tapados (91\%) 


\section{Conclusiones}

- Tener en cuenta métodos, situaciones, contenidos y desempeños que reconozcan las necesidades educativas de la población con discapacidad visual y de esta forma promover su desarrollo mediante el uso de diversas estrategias didácticas que favorezcan un aprendizaje significativo y relevante del pensamiento geométrico.

- Propiciar experiencias de aprendizaje ameno, que reconozca la diversidad dentro del aula de matemáticas y de esta forma logra un aprendizaje significativo.

- El estudiante invidente se puede integrar en una clase frecuente de geometría, si se hacen las adaptaciones curriculares necesarias.

\section{Referencias}

Alsina, C., J. Fortuny, R. Pérez (1997), ¿Por qué Geometría? Propuestas didácticas para la ESO, Madrid: Síntesis.

Deobold, B., Van, D., \& Meyer, W. (2006).

Elliott, John (1996). El cambio educativo desde la investigación-acción. Ediciones Morata, Madrid.
Forte, M., \& Garcia, J. (s.f.). scribd. Recuperado el 15 de mayo de 2013, de El aprendizaje del niño ciego en la escuela: http://es.scribd. com/doc/33070465/APRENDIZAJE-DELNINO-CIEGO-EN-LA-ESCUELA

Godino, J. D., \& Batanero, C. (2003). Fundamentos de la enseñanza y el aprendizaje de las matemáticas para maestros. Madrid: Matemáticas y su didáctica para maestros .

HEGARTY, S. (1994): Educación de niños y jóvenes con discapacidades. Principios y prácticas. Publicaciones de la UNESCO.

MEN. (1998). Lineamientos curriculares para el área de matemáticas. Bogotá: Magisterio.

MEN. (2004). Pensamiento Geométrico y computación. Bogotá: Ministerio de Educación Nacional.

MEN. (2006). Estándares curriculares para el área de matemáticas. Bogotá: Ministerio de Educación Nacional.

SEVILLA, E. D. (s.f.). Guía para la atención educativa a los alumnos y alumnas con déficit visual. Sevilla: Consejería de Educación y Ciencia.

UNESCO (1995): Conferencia mundial sobre necesidades educativas especiales. Salamanca: UNESCO-Ministerio de Educación y Ciencia 